\title{
Acute lymphoblastic leukemia, the Indian scenario
}

\begin{abstract}
Acute lymphoblastic leukemia is the most common leukemia in children. There have been advancements in management of this disease, and still research is on to improve further outcome. A prospective observational study was conducted in a tertiary care hospital in north India from 2008 till 2013, enrolled 186 pediatric acute lymphoblastic leukemia patients.
\end{abstract}

Aims: To see clinical profile of pediatric ALL patients, and to see correlation of day 8 and day 33 blast count with cytogenetic and its effect on overall survival of our patients.

Methods: It was a hospital based observational prospective study. Patients were enrolled from June 2008 till March 2010, after risk stratification, patients received risk adopted BFM 95 protocol, last patient finished his maintenance on August 2013, and study was closed in November 2013

Results: Median age of presentation was 6.5 years and male to female ratio was 3.5:1. High risk disease was seen in $55.85 \%$ patients, Fluorescence In-Situ hybridization (FISH) for TEL AML, MLL, BCR-ABL gene rearrangements were positive in 33.3\%, 9.1\%, 9.7\% patients respectively. Bone marrow was the commonest site of relapse. Day 8 peripheral blood blasts and day 33 bone marrow blasts had no significance as all the patients had complete clearance. So in our study the overall survival at 6 years of analysis was $86 \%$ and event free survival for the entire series was $82 \%$ at 6 years.

Keywords: overall survival efs-event free survival, acute lymphoblastic leukemia, fluorescence in-situ hybridization, cerebrospinal fluid, central nervous system, berlinfrankfurt-munster

\author{
Volume 5 Issue I - 2018

\section{Faisal R Guru,' Javvid Muzamil, ${ }^{2}$ Shumail} \\ Bashir, ${ }^{3}$ Amita Mahajan ${ }^{4}$ \\ 'Registrar Medical Oncology; Sheri Kashmir Institute of Medical \\ Sciences, India \\ ${ }^{2}$ Department of Medical oncology, Sher-i-Kashmir institute of \\ medical sciences, India \\ ${ }^{3} \mathrm{MD}$ chest medicine; Lecturer chest medicine Govt. Medical \\ College, India \\ ${ }^{4}$ Senior consultant in pediatric oncology and hematology at \\ Indraprastha Apollo hospital, India
}

\author{
Correspondence: Javvid Muzamil, Department of Medical \\ oncology, Sher-i-Kashmir Institute of Medical Sciences, India, Tel \\ 9858766055,Email javvidmd@gmail.com
}

Received: February 13, 2018 | Published: April 16, 2018
Abbreviations: OS, Overall survival; EFS, event free survival; ALL, acute lymphoblastic leukemia; FISH, fluorescence In-Situ hybridization; CSF, cerebrospinal fluid; CNS, central nervous system, BFM, berlin-frankfurt-munster

\section{Introduction}

ALL is the most common malignancy in children. ${ }^{1}$ It accounts for $25 \%$ of all childhood cancers and approximately $75 \%$ of all cases of childhood leukemia. ${ }^{2}$ Pediatric ALL is often cited as one of the true success stories of modern medicine. The cure rates have improved from virtually zero, prior to the advent of modern chemotherapy and radiotherapy (in 1950s), to the current overall event-free survival rates of approximately $80 \% .^{2-4}$ The cure rates of children with ALL in India are improving, akin to the western world. However, reports from several institutions in India suggest that outcome is inferior to that achieved in the west. This is not merely due to deaths secondary to toxicity or febrile neutropenia, but because of increased incidence of relapse as well. Etiology for higher incidence of relapsed disease is uncertain. A number of studies from India have indicated that there are a higher proportion of high risk factors in our patients. Though there is limited data on Cytogenetics from India, it suggests that we have a higher proportion of children with unfavorable Cytogenetics or a lower proportion with favorable Cytogenetics. Despite advances in the treatment, 20 to $30 \%$ of children with ALL in whom remission is achieved after initial induction chemotherapy, subsequently relapse. An array of clinical and biological features have been identified as prognostically significant in childhood ALL, including age, presenting leukocyte count, Immunophenotypic, chromosomal abnormalities, the presence of overt central nervous system leukemia and the rapidity with which the patients demonstrate a response to initial induction chemotherapy. ${ }^{4-6}$ Risk factors are utilized for stratification of therapy. ${ }^{6-8}$ More intensive therapies are administered to patients considered to have the highest risk of relapse. In contrast, some of the more morbid components of the therapy are modified or eliminated for children considered to have the best prognosis. The goal of risk adapted therapy is to "treat away" adverse presenting features so that cure rates are similar for patients with high risk and low risk disease. ${ }^{9,10}$ There was a need to assess survival data and identify risk factors for relapse in our set of patients. Although there are multiple studies performed across the world, there is paucity of literature available on the risk factors, pattern of relapsed disease and outcome of children with ALL in India. ${ }^{4-6}$ With this background we conducted a study to assess the various prognostic factors and the impact they have on the outcome in children treated for ALL in our institute focusing on Immunophenotypic and Cytogenetics

\section{Aims and objectives}

a. Immunophenotypic and cytogenetic profile in pediatric acute lymphoblastic leukemia patients.

b. Correlation of Cytogenetics and Immunophenotypic with day 8 peripheral blood blast counts and day 33 marrow remission status during induction phase 1.

c. Correlation of Cytogenetics and Immunophenotypic with overall survival in our patient cohort.

\section{Methods}

The study was a hospital based, observational study conducted 
between June 2008 to November 2013. The study included analysis of data of children in the age group of 1 to 18 years, diagnosed as acute lymphoblastic leukemia (ALL), who visited the Apollo Centre for Advanced Pediatrics, Indraprastha Apollo hospital during the study period for their treatment. All the cases of ALL below age of 1 year (infant ALL) and those who had already relapsed at the time they presented to our centre were excluded from the study. The data was collected from 186 patients regarding their age, gender, laboratory indices like hemoglobulin, leukocyte count, platelet count, CSF cytology (positive or negative for blasts), Immunophenotypic data (Pre B ALL, B cell ALL, T cell ALL) by flow cytometry, Cytogenetics data (hypodiploidy, normal, hypodiploidy) by conventional karyotyping method) and in a few patients Fluorescent In Situ Hybridization (F.I.S.H) technique was used to determine presence or absence of BCR-ABL, TEL AML, MLL gene rearrangements. In the 186 patients that opted for treatment the status of blasts in peripheral blood at day 8 and bone marrow status at day 33 was assessed to determine the response to therapy. The outcome in terms of survivor and nonsurvivor was determined. The last point of contact in survivors and non-survivors was recorded. All the patients were risk stratified and the treatment was modified accordingly. Treatment protocol used was modified BFM 95 protocol, and treatment was risk adopted, either standard or high risk. Analysis of disease outcome was examined as overall survival (OS) and event free survival (EFS). OS was measured from date of initial diagnosis of ALL to date of death from any cause or date of last contact using the Kaplan-Meier method which is a nonparametric (actuarial) technique for estimating time related events (the survivorship function).

\section{Results and observation}

This study was prospective observational in nature. The first patient was enrolled in June 2008 and the last patient was enrolled in March 2010. All patients had completed the risk adopted protocol including maintenance, and last maintenance finished in August 2013. The data of 186 patients with ALL who visited our Centre during the study period revealed the following results. The age range of the study population varied between 1.5 to 15.8 years. The mean and the median age were $7.2( \pm 4.1)$ and 6.5 years respectively. Majority of patients $(74.6 \%)$ patients were below 10 years (Figure 1A). There were $78.1 \%$ boys and $21.9 \%$ girls (Figure $1 \mathrm{~B}$ ). The male: female ratio was 3.5:1. Central nervous system disease at diagnosis was seen in only 4 patients and they all received cranial irradiation. The Total leukocyte count (TLC/ $\mu \mathrm{l})$ ranged between 600 to 7lakhs. The mean and the median TLC were 39000 and 8300 respectively. The TLC was more than 50000 in $23.7 \%$ patients (Figure $2 \mathrm{~A}$ ). The platelet count at presentation was less than $30.0 \times 10^{9} / \mathrm{L}$ in $18.4 \%$ patients, more than $30.0 \times 10^{9} / \mathrm{L}$ to $50.0 \times 10^{9} / \mathrm{L}$ in $50 \%$ patients and greater than $50 \times 10^{9} / \mathrm{L}$ in $31.6 \%$ patients (Figure $2 \mathrm{~B}$ ). Risk stratification of the patients has been done in this study into high risk, standard risk and treatment was modified accordingly, giving more intense therapy to the high risk patients. Patients having any one of the following criteria like Age $>10$ years, TLC $>50,000 / \mu 1$, CNS disease present at diagnosis, immuno phenotyping showing $\mathrm{T}$ cell ALL, cytogenetic analysis showing hypodiploidy or MLL gene rearrangement or BCR $\mathrm{ABL}$ arrangement was considered high risk and the rest as standard risk. Analysis revealed that $104(55.85 \%)$ patients were high risk and the remaining $82(44.14 \%)$ patients were standard risk. The immunephenotyping analysis revealed Pre B ALL in $88.6 \%$, B cell ALL in $1.7 \%$ and $\mathrm{T}$ cell ALL in $9.7 \%$ patients (Figure $3 \mathrm{~A}$ ). Conventional cytogenetic revealed normal cytogenetics in $78 \%$, hypodiploidy in $9.5 \%$, hypodiploidy in $4.8 \%$, and $7.5 \%$ failed to culture (Figure $3 \mathrm{~B}$ ). FISH for TEL AML, MLL, BCR-ABL gene rearrangement analysis was positive in $33.3 \%, 9.1 \%, 9.7 \%$ patients respectively, with $48 \%$ had no FISH available (Figure 4A-C).

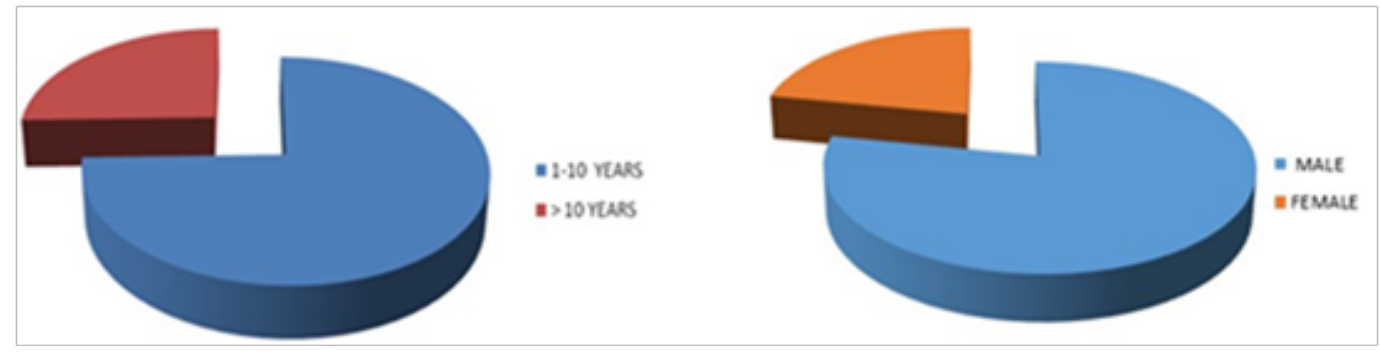

Figure I (A) Around 25\% patients above 10 years of age, and rests were below 10 years. (B) More than $2 / 3^{\text {rd }}$ was boys and male to female ratio was $3.5: 1$.

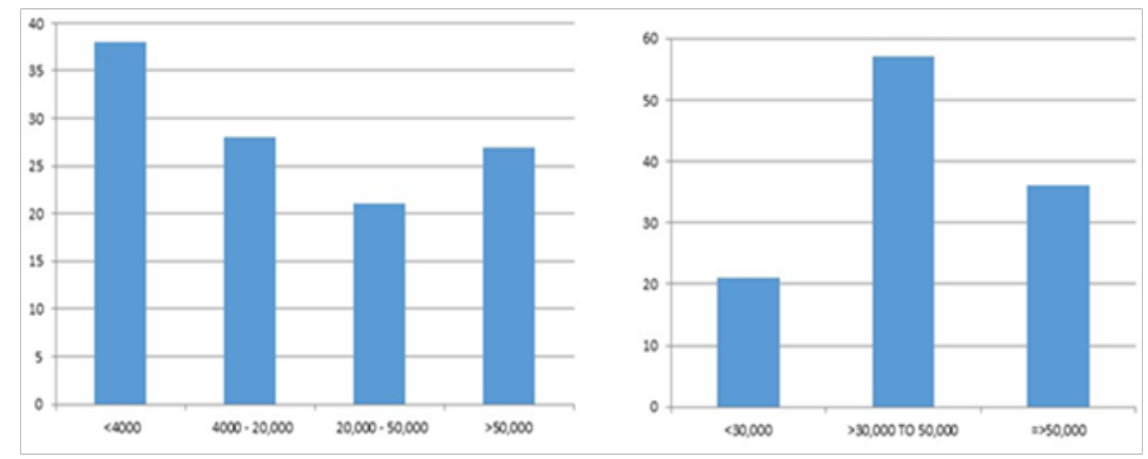

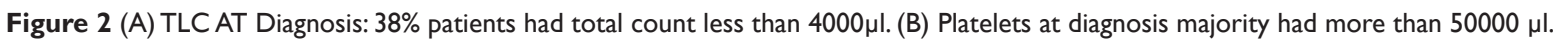




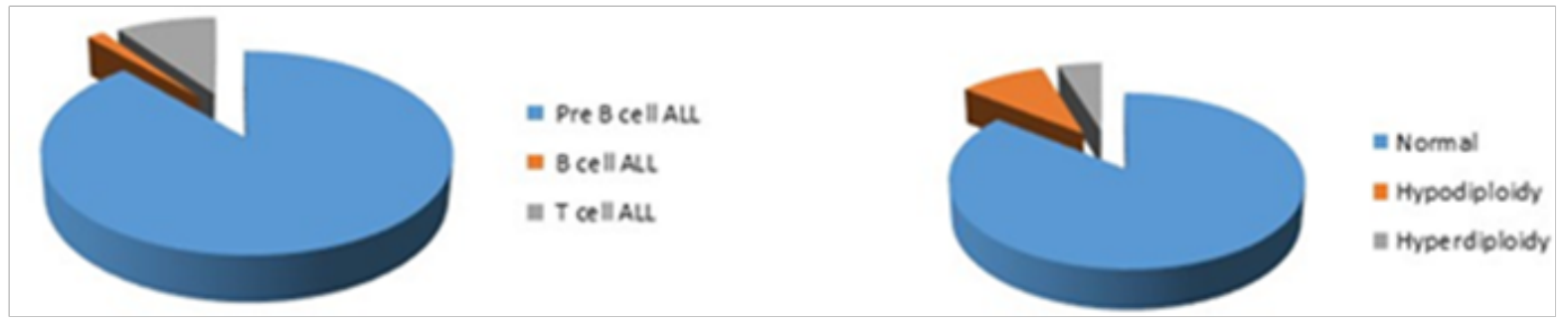

Figure 3 (A) Immuno-phenotyping, pre B cell $88.6 \%$,T cell is $9.7 \%$. (B) Conventional Karyo type normal Cytogenetics $78 \%$, Hypodiploidy in $9.5 \%$
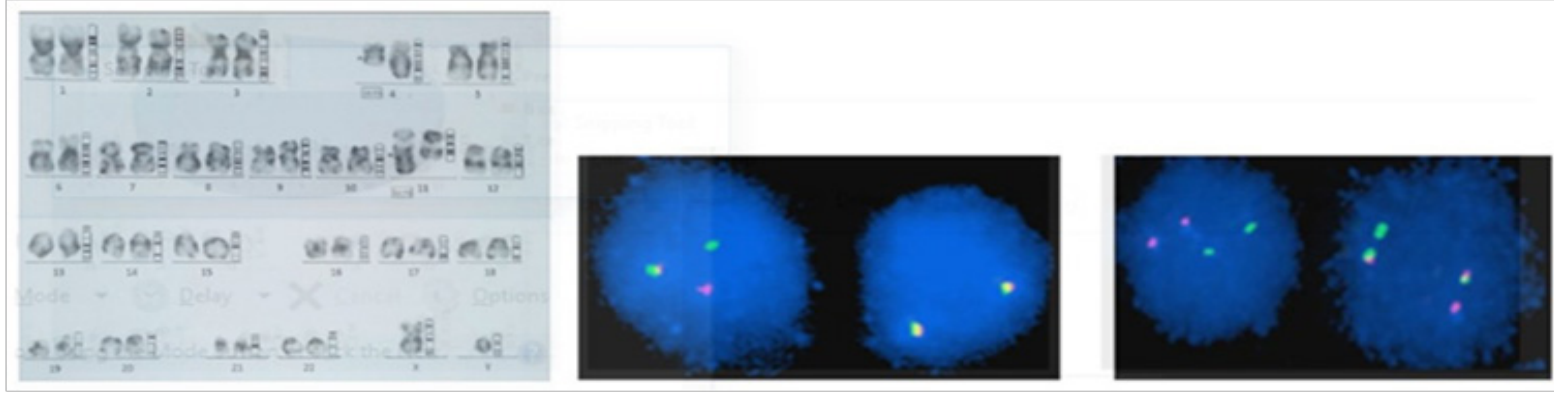

Figure 4 (A) Conventional Karyogram showing Conventional Karyogram showing t (4-II) in a patient. (B) MLL gene rearrangement by FISH in one of our patient. (C) BCR ABL positive by FISH in one of our patient.

All the study patients received treatment. The treatment was risk stratified on the basis of few parameters listed above. We had high risk patients (56\%) more than standard risk (44\%). So, treatment was instituted as either high risk BFM 95 protocol or Standard risk BFM 95 protocol of the 186 patients who received therapy, the peripheral blood status at day 8 showed complete disappearance of blasts in all the patients. The bone marrow status at day 33 revealed complete remission in 184 patients since there were 2 induction deaths. The outcome of 186 patients revealed 161 survivors and 25 non survivors. Among the 25 non survivors, 17 patients had either relapsed or died, 7 patients died due to treatment related toxicity out of which 2 were induction deaths and 1 patient died in an accident. Bone marrow was the commonest site of relapse. So in our study day 8 peripheral blood blasts and day 33 bone marrow blasts had no significance as all the patients had complete clearance. So in our study the overall survival at 6 years of analysis was $86 \%$. The overall survival (in months) of patient's range was in the range of 1 month to 87 months with mean of $31.32( \pm$ SD 20.5$)$ and median of $28.00( \pm 3.8)$. The estimated mean survival time for the high risk and standard risk patients were
$58.41 \pm 5.6$ and $74.88 \pm 8.4$ months respectively and this difference was not statistically significant $(\mathrm{p}=0.896)$ (Figure $5 \mathrm{~A})$. The estimated OS for the entire series at 6 years was $86 \%$. The EFS for the entire series was $82 \%$ at 6 years (Figure 5B). The median survival time for males and that for females was similar and the difference was not statistically significant $(p=0.267)$. The median survival time of the patients who had CNS disease at diagnosis was similar to those who did not and the difference was not statistically significance $(\mathrm{p}=0.351)$. The median survival for patients with TLC $<50.0 \times 10^{9} / \mathrm{L}$ and for those with TLC $>50.0 \times 10^{9} / \mathrm{L}$ was similar and was not statistically significant $(\mathrm{p}=.242)$. The difference in the median survival time for patients with Pre B ALL and T cell ALL was not statistically significant. The median survival time in patients, who had normal Cytogenetics, hypodiploidy and hypodiploidy were similar and this difference was statistically not significant. $(\mathrm{p}=.263)$. Among patients who got relapse, they belonged to all subsets, viz, Normal Cytogenetics, ABL- BCR, TEL- AMLor MLL abnormalities (Figure 6A) (Figure 6B). The survival difference was insignificant among these groups.

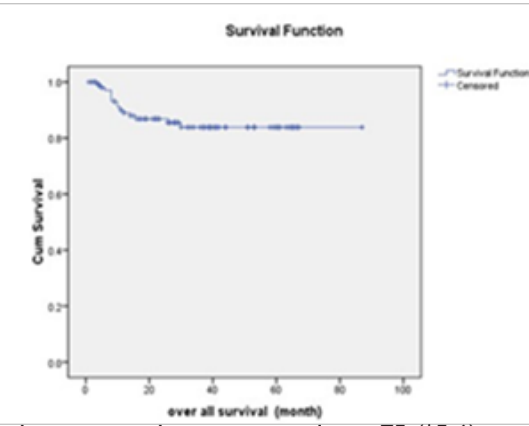

Figure 5 (A) Mean survival, the estimated mean survival was $75(* 5.6)$ months. groups was not statically significant $\left(p^{*} 0.583\right)$.

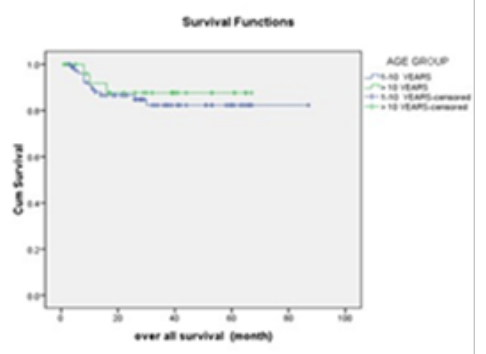

(B) Mean survival as a function of age, the different in estimated OS in the 2 age 


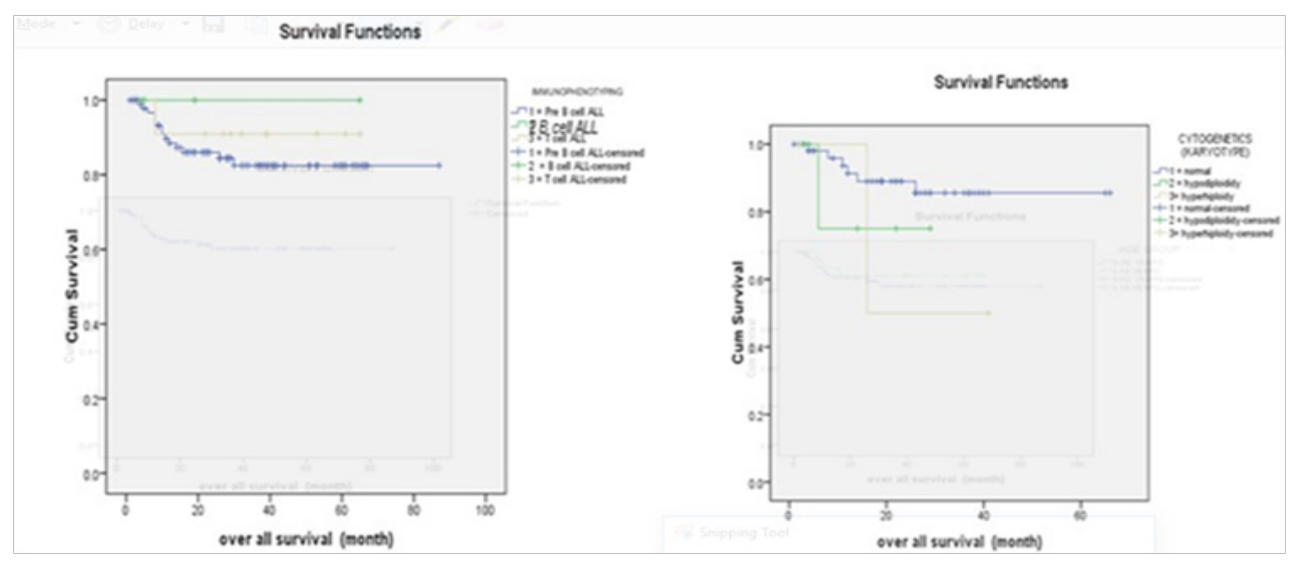

Figure 6 Overall survivals as a function of immune phenotype, higher in B cell patients, with no statistical significance. (B) Overall survival in months as per cytogenetic, higher in normal karyotype patients $(58.47 \pm 5.6)$ with no statistical significance.

\section{Discussion}

In our study population Pre B ALL was seen in $88.6 \%$, B cell ALL in $1.7 \%$ and $\mathrm{T}$ cell ALL in $9.7 \%$ patients. In our study we found that all the B cell ALL patients were alive at the time of analysis and the difference in outcome in terms of overall survival between Pre B cell ALL and T cell ALL was not statistically significant. In our study treatment was stratified with T cell ALL classified as high risk and receiving more intensified treatment. In the study by Goldberg et al. ${ }^{11}$ from the Dana Farber Cancer Institute (DFCI), analyzed the outcome of 125 patients with T cell ALL found no significant difference in EFS comparing patients with T-ALL and B-progenitor ALL, although T-ALL patients had significantly higher rates of induction failure, and central nervous system (CNS) relapse. In the COALL studies T-ALL had a similar prognosis as B-precursor ALL except in COALL-89, in which only a small number of patients was entered. ${ }^{12}$ In two studies by Chessells JM et al. ${ }^{13,14} \mathrm{~T}$ cell ALL was seen to have a worse prognosis and early relapse was more common in children with T-ALL. In a study by Horibe et al. ${ }^{13}$ found that incidence of T-cell ALL to be less as compared to those reported by Tokai and Hokkaido in Kansai and Chu-Shikoku. Patients with B-cell precursor (B-pre) ALL had a more favorable prognosis as compared to T-cell ALL patients. ${ }^{14}$ In the analysis of published data comparing the prognostic determinants in childhood ALL in Negroid and Caucasian population, Macharia $\mathrm{WM}^{15}$ found the proportions for $\mathrm{T}$ cell Immunophenotypic in developed countries and developing countries to be $17 \%$ and $60 \%$ respectively. In a study by Hussein et al. ${ }^{7} \mathrm{~B}$-cell precursor phenotype was encountered in $73.4 \%$ of patients, T-cell in $26.6 \%$. In our study, Conventional Cytogenetics revealed normal Cytogenetics in $78 \%$, hypodiploidy in $9.5 \%$, hypodiploidy in $4.8 \%$, and $7.5 \%$ failed to culture (Figure 3B). The median survival time in patients, who had normal Cytogenetics, hypodiploidy and hypodiploidy were similar and this difference was statistically not significant $(\mathrm{p}=.263)$.

FISH for TEL- AML, MLL, BCR-ABL gene rearrangement analysis was positive in $33.3 \%, 9.1 \%, 9.7 \%$ patients respectively, with $48 \%$ had no FISH available. In our study we risk stratified treatment with hypodiploidy, MLL or BCR ABL gene rearrangements, considered as high risk and received more intense therapy The difference in the median survival time among patients with normal Cytogenetics, hypodiploidy and hypodiploidy was statistically not significant $(\mathrm{p}=.263)$. In a study by Secker-Walker et al. ${ }^{16}$ found that $17(21 \%)$ patients with high hypodiploidy tended to have low leukocyte counts and common ALL, but their favorable outcome $(75 \%$ event-free survival) was independent of these factors. No patient with hypodiploidy survived while the pseudo diploid and normal groups had an intermediate prognosis. In a study by Horibe et al. ${ }^{14}$ found that presence of the Philadelphia chromosome, and translocations associated with chromosome 11q23 as an unfavorable factor for EFS. Hypodiploidy ( $>50$ chromosomes) was significantly favorable for EFS. In a study by Kebriae P et al. ${ }^{9}$ found hypodiploidy in $45 \%$, normal karyotype in $9 \%$, hypodiploidy in $6 \%$ and pseudodiploidy in $40 \%$. Since in our study the individual groups had similar median survival and it had no statistical significance. The study conducted by K.S Tsang et al., ${ }^{16}$ showed TEL/AML rearrangements had better induction response and longer complete remission. In a study by $\mathrm{D}$ Sugapriya et al., ${ }^{17}$ ABL-BCR positivity was seen in $8.3 \%$ patients in southern India, which was $9.7 \%$ in our study. Long-term survival rates in pediatric ALL with BCR-ABL positive is around $80 \%$, which drops down to $30 \%$ in adults, with $86 \%$ overall survival at 6 years in our study. ${ }^{1}$ Similarly MLL rearrangements has worse outcome in adult ALL than in childhood. ${ }^{18}$

\section{Conclusion}

The study has evaluated the survival outcome in children with ALL and has determined the prognostic factors like Immunophenotypic, Cytogenetics in addition to conventional factors associated with adverse outcome and survival rates in a large cohort of patients in the Indian population. The study clearly shows that the prevalence of adverse prognostic factors is not very high in the Indian population as had been previously reported in various Indian studies. The results and outcome of this study have been compared with the results from the previous studies from India and those from the developed countries. This study clearly shows that with appropriate risk stratification of patients with adverse prognostic factors and treatment modification accordingly we can get the outcomes in our setting which is comparable to that in the western world.

\section{Acknowledgements}

None. 


\section{Conflict of interest}

The author declares there is no conflict of interest.

\section{References}

1. Pui CH, Evans WE. Acute lymphoblastic leukemia. $N$ Engl J Med. 1998;339(9):605-615.

2. Gaynon PS. Childhood acute lymphoblastic leukemia and relapse. $B r J$ Haematol. 2005;131(5):579-587.

3. Chessells JM, Hardisty RM, Richards S. Long survival in childhood lymphoblastic leukaemia. Br J Cancer. 1987;55:315-319.

4. Shanta V, Maitreyan V, Sagar TG, et al. Prognostic variables and survival in pediatric acute lymphoblastic leukemias: Cancer Institute experience. Pediatr Hematol Oncol. 1996;13(3):205-216.

5. Vaidya SJ, Advani SH, Pai SK, et al. Survival of childhood acute lymphoblastic leukemia: results of therapy at Tata Memorial Hospital, Bombay, India. Leuk Lymphoma. 1996;20(3-4):311-315.

6. Magrath I, Shanta V, Advani S, et al. Treatment of acute lymphoblastic leukaemia in countries with limited resources; lessons from use of a single protocol in India over a twenty year period. Eur $J$ Cancer. 2005;41(1):1570-1583.

7. Hussein H, Sidhom I, Naga SA, et al. Outcome and prognostic factors of acute lymphoblastic leukemia in children at the National Cancer Institute, Egypt. J Pediatr Hematol Oncol. 2004;26(8):507-514

8. Secker-Walker LM, Chessells JM, Stewart EL, et al. Chromosomes and other prognostic factors in acute lymphoblastic leukaemia: a long-term follow-up. Br J Haematol. 1989;72(3):336-342.

9. PadmanjaIi KS, Gupta S, Tomar S, et al. Relapse pattern of acute lymphoblastic leukemia in children treated on an uniform regimen and analysis of risk factors. Indian JPedia tr. 2002;69:S2-S8.
10. Amare P, Gladstone B, Varghese C, et al. Clinical significance of cytogenetic findings at diagnosis and in remission in childhood and adult acute lymphoblastic leukemia: experience from India. Cancer Genet Cytogenet. 1999;110(1):44-53.

11. Goldberg JM, Silverman LB, Levy DE. Childhood T-cell acute lymphoblastic leukemia: the Dana-Farber Cancer Institute acute lymphoblastic leukemia consortium experience. $J$ Clin Oncol. 2003;21(9):3616-3622.

12. Harms DO, Janka-Schaub GE. Co-operative study group for childhood acute lymphoblastic leukemia (COALL): long-term follow-up of trials 82, 85, 89 and 92. Leukemia. 14(2):2234-2239.

13. Chessells JM, Veys P, Kempski H, et al. Long-term follow-up of relapsed childhood acute lymphoblastic leukaemia. $\mathrm{Br} J$ Haematol. 2003;123(3):396-405.

14. Horibe K, Hara J, Yagi K, et al. Prognostic factors in childhood acute lymphoblastic leukemia in Japan. Int J Hematol. 2000;72(1):61-68.

15. Macharia WM. Comparison of prognostic determinants in childhood acute lymphoblastic leukemia in Negroid and Caucasian populations. East Afr Med J. 1996;73(10):638-642.

16. Tsang KS, Li CK, Chik KW, et al. TEL/AML1 rearrangement and the prognostic significance in childhood acute lymphoblastic leukemia in hong kong. American Journal of Hematology. 2001;68(2):91-98.

17. Sugapriya D, Preethi S, Shanthi P, et al, $B C R-A B L$ translocation in pediatric acute lymphoblastic leukemia in southern india. Indian $J$ Hematol Blood Transfus. 2012;28(1):37-41.

18. J-Q Mi, Wang X, Yao Y, et al. Newly diagnosed acute lymphoblastic leukemia in China (II): prognosis related to genetic abnormalities in a series of 1091 cases. Leukemia. 2012;26(7):1507-1516. 